\title{
Study on the rights and interests of subject in clinical trials
}

\author{
Zhang Shu ${ }^{1}$, Zhu Dongyun ${ }^{1}$, Qin Jie ${ }^{1^{*}}$ \\ ${ }^{1}$ School of Public Affairs, University of Science \& Technology of China, 230026,P.R.China.
}

\begin{abstract}
Clinical trials are essential measures to determine the efficacy and safety of drugs. Clinical trials of new drugs and new medical devices have also received strong support at the national level. In the case of more and more clinical trial cases, how to better protect the rights of the subject becomes a problem that needs to be discussed in more depth. Based on the background of the development of clinical trials, this paper describes the lack of rights protection under the traditional support methods of clinical trial subject and then analyzes the problems of the subject's right to life, body autonomy, informed consent, privacy and compensation. Problems with current subject rights protection and possible safeguards. The paper believes that the development and application of new life technologies make the clinical trial process more complicated and the results more uncertain. Therefore, the protection of traditional subject rights is not perfect, we need to expand legislative coverage, improve the rights dimension of subject rights protection and the structural dimension analysis of the ethics review committee which are effective measures to protect the rights of subject.
\end{abstract}

Keywords: clinical trial, subject rights, rights protection

DOI: $10.7176 /$ PPAR/9-12-01

Publication date: December $31^{\text {st }} 2019$

\section{Research background}

In October 2017, the report of the 19th National Congress of the Communist Party of China clearly put forward the "implementation of the healthy China strategy", emphasizing that "people's health is an important symbol of national prosperity and national prosperity", and health began to integrate into all fields and all aspects of work. In order to better cooperate with China's health strategy, in 2018, the Executive Council of the State Council decided to develop "Internet + medical health" measures, and put forward "to change the application of clinical trials from the approval system to the default system when expire", and open wider field policy channels for clinical trial behavior. The pilot of the clinical trial hospital is the first, which provides the necessary conditions for some areas to explore the development path of the trial hospital. For example, the Beijing municipal government issued the Beijing action plan for accelerating collaborative innovation in medical and health care (2018-2020), emphasizing the joint construction mode of medical institutions, colleges and universities, scientific research institutes and enterprises, introducing international operation and management team, pilot the construction of independent clinical trial hospitals, and actively explore sustainable operation mechanism. On November 7 , the website of Shanghai municipal government released the implementation opinions on deepening the reform of the review and approval system and encouraging the innovation of pharmaceutical medical devices (after this referred to as the opinions). For promoting the development of clinical trials, an essential link in the process of drug research and development, Shanghai made more specific regulations on the reform of clinical trial management, which is also the focus of drug approval system reform.

Clinical trials cost a lot of money and time. Clinical trials in China started late, and clinical trials are in a rapid development stage. At present, large hospitals have clinical trial centers. By the middle of March 2019, there are 646 qualified clinical trial institutions in China, including Guangdong, Beijing, Jiangsu, Shanghai and Shandong. The top five provinces, the number of qualified clinical trial institutions accounting for $40 \%$ of the country, which is positively related to population distribution, economic development level and medical and health resources. The clinical trial hospital is different from the conventional hospital. It is an exploratory operation through the way of policy concession and trial first, so as to reduce the examination and approval as much as possible, support new therapy, new drugs and new equipment and materials to collect a small number of patients to carry out the trial, and is an important platform integrating basic research, clinical trials and medical services ${ }^{[1]}$.

From the existing policies and the development trend of reality, the clinical trial behavior of new drugs and new medical devices has been strongly supported by the national level, and more and more regions begin to work in clinical trial hospitals. Some regions, such as Beijing, have formulated policies for medical institutions to adapt to the development of clinical trial hospitals, explore the combination of new technologies and traditional breakthrough innovation in terms of fundamental principles, development goals and critical tasks.

Because of the particularity of the process and the uncertainty of the results, the management of clinical trials is very complicated. The development of new life science and technology improves people's health level and promotes the increase of drug clinical trials. For patients' subject, they can understand the advanced treatment level and methods for their diseases, and get free drug treatment, reduce pain and pressure, and get the chance to cure ${ }^{[2]}$. However, the risk of participating in the clinical trial should not be underestimated. Although the clinical trial needs to be approved by the National Drug Administration and the process is rigorous and safe, it can not be ruled out that the treatment may not be practical, or even 
there may be some serious or even life-threatening effects, and the subject of the clinical trial need more time and energy to participate in the overall Clinical trial process ${ }^{[3]}$. Therefore, to respect and protect the legitimate rights and interests of the subject is the primary problem to be solved in clinical trials. So, under the existing system, how to protect the rights and interests of the subject? According to the "drug clinical trial quality management standard" the rights, safety and health of subject must be higher than the consideration of scientific and social interests. In order to adequately protect the rights and interests of the subject, the existing protective measures are that the clinical trials must be reviewed and approved by the ethics committee, and the subject must sign the informed consent.

With the development of life science and technology, the traditional measures to protect the rights and interests of subject have been unable to cope with the complicated process and high-risk results of clinical trials, while the demand and actual volume of clinical trials are increasing. According to the drug clinical trial registration and information publicity platform, as of September 26, 2019, the total number of trials registered on the platform was 12150, and the annual registered clinical trials increased from 916 in 2014 to 2570 in 2018, of which only $9.55 \%$ were traditional Chinese medicine and natural drugs with less human response, $17.6 \%$ were biological products, and $72.85 \%$ were chemical drugs with more significant human response. According to the statistical data of clinical trials of medical devices obtained by the drug intelligence data network, as of September 26, 2019, the number of registered clinical trials of medical devices is 315460 . On one hand, these data reflect China's independent innovation on advanced drugs and medical devices as well as its absorption, introduction and re innovation, all of which have made progress. However, at the same time, the development of life technology and medical technology will also bring more new and complex legal issues. Although the traditional management of clinical trials can partially protect the rights and personality of the subject, there is still a long way to go in terms of the legal rules of clinical trials and the protection of the rights and interests of the subject, whether compared with the current situation or the developed countries ${ }^{[4]}$. Therefore, there is positive practical significance to explore the protection of the rights and interests of clinical trial subject from the perspective of law.

\section{Rights and interests of clinical trial subject}

The drugs or devices in clinical trials are not on the market yet. According to the data described above, in recent years, the number of clinical trials and subject has been increasing. What is a clinical trial? According to the "quality management standard for clinical trials of drugs" issued by the national drug regulatory authority, clinical trials of drugs refer to the tests on the effects, adverse reactions, absorption and metabolism of drugs in the body of patients or healthy volunteers. The ultimate purpose is to study the efficacy and safety of drugs. For this concept, it is understood from three important aspects: first, the subject include patients or healthy volunteers; second, the purpose of the trial is the efficacy and safety of the drug; finally, the process of the trial is the reaction and role of the drug in the human body. To achieve the purpose of final verification, it needs to go through the initial safety evaluation and the fourth stage of the independent application of the applicant ${ }^{[5]}$.

The subject invited or actively participated in the trial are collectively referred to as "subject". For the subject of patients, they can learn about the advanced treatment level and methods for their diseases, and get free drug treatment, relieve pain and pressure, and get the chance to cure.

Because the safety and effectiveness of these unlisted drugs or devices are still uncertain, the biggest difference between the subject of clinical trials and patients with general medical behaviors is that the subject of clinical trials have greater risks and uncertainties, which are closely related to the life health and personality of the subject of clinical trials. Therefore, the most important thing is to protect the rights and interests of the subject.

\subsection{Subject's right to life and health}

The right to life and health is the basis for natural persons to participate in all social activities and enjoy all other rights. It is the most fundamental right and the most basic human rights. Life is the highest personal interest of the natural person and the basis of other personal rights and interests. Article 110 of the 《General Principles of Civil law》 stipulates that natural persons have the rights of life, body, health, name, portrait, reputation, honor, privacy, marriage autonomy and so on. In practice, it is more common for subject in clinical trials to participate in the trial project, take the drugs or use the test equipment in the trial, resulting in various side effects and adverse reactions, resulting in the impairment of the right to life and health ${ }^{[6]}$. Clinical trials take people as subject, and the right to life and health of subject is the most basic and important.

\subsection{Subject's physical autonomy}

First of all, in the clinical trials of human medicine, the stand is different. The interests of the experimenter are not always in line with those of the experimenter. Even when there is a conflict of interest, it will harm the rights and interests of the experimenter. Therefore, for the experimenter, they cannot be required to give full care like doctors to patients. In order to better protect the rights of the subject, more and more emphasis is placed on law and ethics Autonomy of subject. Body autonomy is a person's right to manage and claim his body and mind. In clinical trials, the right of body autonomy not only includes the right of subject to control their own body freely, but also includes psychological independence, and they are the best masters without interference from others. When the subject participate in the clinical trial, they have the right to decide 
when to join the clinical trial, and also have the right to decide when to withdraw from the trial. For example, in the process of the trial, there is a potential risk or has caused physical damage to the subject, the subject request to withdraw from the trial, and the trial party is not forced to continue, and the withdrawal behavior does not affect the other medical treatment rights of the subject. Of course, any freedom is limited, on the premise of not violating the law and endangering public social morality, anyone has the right to decide his affairs or even life ${ }^{[7]}$.

\subsection{Subject's right to privacy}

The right of privacy generally refers to the right that a natural person enjoys private information and outsiders do not know personal secrets. Everyone pays great attention to their privacy and needs to protect their privacy. The right to privacy is stipulated in the tort liability law and the quality management standard of drug clinical trials. In clinical trials, the trial party can obtain more comprehensive personal information, such as a home address, diagnosis, and treatment records, contact information, family history, identity information and other contents. The detailed personal information is easy to quickly determine a person in the vast sea of people ${ }^{[8]}$. In reality, the drugs or devices in clinical trials are mainly aimed at the cases of difficult and miscellaneous diseases or critical diseases. Among these patients, there are inevitably diseases that the subject are not willing to disclose, such as participating in the treatment trials of sexually transmitted diseases or infectious diseases. The behaviors they participate in are to publicize the contents of the patients suffering from this disease. Of course, not all medical information is personal privacy, only the medical information that can reflect or involve personal privacy belongs to the scope of non disclosure. Therefore, the subject have the right to ask the sponsor and the experimenter to protect their privacy within the scope of law.

\subsection{Subject's right to informed consent}

The right of informed consent is an important ethical right derived from the subject's body autonomy. The right of informed consent in medical clinical trials refers to the right of subject to voluntarily choose to participate in, not participate in or withdraw from clinical trials when they fully know and understand the purpose, content and risk of the trials. Informed consent is one of the important ways for the subject to understand the clinical trial projects they participate in. The effective carrier of informed consent is informed consent. Whether the trial party informs the trial projects completely, comprehensively and objectively will seriously affect the subject' important decisions on whether to participate in or withdraw from the trial and understanding of the trial risks. The right of informed consent requires the subject to fully understand the project, nature, risk, compensation method and other information of the clinical trial, and on this basis, the subject voluntarily express their consent ${ }^{[9]}$. The right of informed consent requires two parts: informed consent and informed consent. Informed consent is the premise, but consent is not an inevitable result. Knowing requires the subject to have certain cognitive or behavioral abilities. When the subject fully understand the contents of the test project, they can decide whether to participate or withdraw from the test. In this regard, we should also give full consideration to the personal guarantee of the right of informed consent of special groups such as children, people with mental health and so on. Due to the late start of clinical trials in China, the general public does not know much about the clinical trials, and the right to informed consent also enables the subject to have a deep understanding of the specific contents of the clinical trials they participate in and the possible risks, so as to reduce the unnecessary fear of the subject and their families caused by the lack of understanding ${ }^{[10]}$. Informed consent is a legal and ethical rule in clinical trials.

\subsection{Subject's right to relief for damage}

In the clinical trial, due to the inevitable risk and individual differences of the subject's physical quality, or the subject's wrong cognition and understanding of the risk of the clinical trial due to many factors such as the level of personal cognition, the sponsor and the trial party shall make corresponding compensation for the damage caused to the subject by the trial party within the scope of the duty of care ${ }^{[11]}$

Meanwhile, if the trial is successful, the whole human society will benefit from it in the long run, while in the short run, the sponsor and the trial party will enjoy the exclusive right and corresponding profit after the trial is successful In case of unequal distribution, the sponsor or the experimenter shall compensate or reward the subject accordingly.

\section{Problems in the protection of subject's rights and interests in clinical trials in China 3.1 Relevant laws and regulations on the protection of subject's rights and interests need to be improved}

As mentioned above, according to the drug clinical trial registration and information publicity platform, as of September 26,2019 , the total number of trials registered on the platform is 12150 , and each trial project needs to recruit tens of people less and tens of thousands of subject more. According to the data, at present, there are more than 800 kinds of clinical trials for new drugs alone in China every year. The number of subject need for these clinical trials is about 500000 every year. The number of domestic clinical trials has increased dramatically. With the increase in the number of clinical trials, there are more and more disputes about the infringement of clinical trials, which brings challenges to law and ethics. However, in reality, only a few cases of infringement of clinical trials are sued in court and are sued by news media The report is known to the public. At present, compared with developed countries, the relevant laws and regulations of clinical trials in China are still in an imperfect stage ${ }^{[12]}$. 


\subsubsection{Relevant laws and regulations are not perfect in legislation}

At present, there is no special clinical trial law in China, which mainly regulates the clinical trial behavior through two administrative regulations, i.e. the quality management standard of drug clinical trial and the ethical review method of biomedical research involving people (Trial). The legal rank is relatively low, and a complete legal system has not yet been formed. Legal responsibility can only be administrative responsibility, but it is also lack of compulsion when implemented. These Regulations are restricted by principle, but they are not clear, and the binding force on the personnel in the clinical trial process can not achieve the corresponding effect ${ }^{[13]}$.

\subsubsection{Lack of independent tort liability for clinical trials}

In terms of liability, when the legitimate rights and interests of the subject are damaged due to the infringement, the tort liability law does not stipulate the tort liability of clinical trials. In the tort cases of clinical trials, there are disputes on the determination of liability, the bearing of liability and the principle of liability attribution, which is not conducive to the legal protection of the rights and interests of the subject. Specifically, in practice, when the court is trying clinical trial cases, it usually applies the general provisions of the tort liability law or the relevant contents of medical damage liability to solve the tort disputes in such cases, and then applies the fault liability in the principle of liability attribution. ${ }^{[14]}$ The subject need to prove the fact of damage and the causal relationship between the behavior and the result, clinical trial Experiments involve very professional medical knowledge and information. The information between the experimenter and the experimenter is not equal in itself. The subject is in a relatively weak position, which leads to the ineffectiveness of proving when the subject suffers from the damage and cannot protect the rights and interests of the experimenter.

\subsubsection{The system of the victim's damage relief is not perfect}

Internationally, the declaration of Helsinki and the international ethics guide for biomedical research on human body stipulate the compensation provisions for subject, but they are not enforced, only constrained from the moral level. From the perspective of our country, although the biomedical ethics review method concerning people stipulates that the sponsor and the trial party shall bear the treatment fee and compensation/compensation for the damage in the clinical trial, it does not mention the scope, amount and procedure of compensation/compensation. In order to avoid the liability risk, the testing party often blurs the definition in the test plan or informed consent. In fact, for many clinical trials funded by the government and scientific research institutions, there is no special compensation/compensation for the subject, only including the necessary follow-up transportation subject. However, in the unfunded clinical research initiated by the trial party, there is almost no special subject compensation / compensation fee. Most clinical trials are only an important part of medical research, and their risks are much higher than ordinary medical behaviors, so it is difficult to ensure that the subject are completely harmless ${ }^{[15]}$. If the sponsor of the trial should compensate for all the damages of the subject, no one may be all the damages of the subject should be compensated by the sponsor of the trial, it may happen that no one is willing to carry out clinical trials for the sake of seeking benefits and avoiding harms, and there will be no new drugs on the market. Ultimately, the interests of all the patients will be damaged, which is not conducive to the healthy development of the pharmaceutical industry. An appropriate damage risk dispersion system and incomplete damage relief system should be established to ensure that the subject can get timely and effective relief when they are damaged. At present, the insurance for clinical trials in China is just starting. The first "liability insurance for clinical trials of drugs" in China is launched by Minsheng insurance company, while other insurance companies are engaged in clinical trials insurance not many. To sum up, these practical factors are not conducive to dispersing the risk of clinical trials.

\subsection{Problems with the protection of the subject's informed consent}

China's existing normative documents for standard clinical trials, the provisions on the subject's right to know are too singular, although the provisions include the content of the notice, the content of the review, because of the different projects of each clinical trial, level, The focus of the notice will vary. In practice, due to the trend of interest, there is a tendency for the tester to evade the key risk information, exaggerating the expected effect, that is, notifying the notification that the informed consent is incomplete ${ }^{[16]}$.

The subject come from all hierarchy. The cultural level, economic basis and medical knowledge reserve of the subject will be different. For example, some subject have a lower cultural level and weak economic foundation. In the case that the professional term of the test item cannot be understood, there is inherently unequal information between the tester and the subject. Compared with the tester, the subject is in a weak position, which does not have the effect of informed notification.

In judicial practice, it is worthy of recognition that the court generally supports the subject's right to informed consent, but usually does not distinguish between the consent of the subject in the clinical trial and the right of informed consent in the general medical dispute. The risks of clinical trials are virtually unknown. Even if these drugs are developed based on existing scientific and technological levels, they have passed animal testing, but their risk is still much higher than that of already marketed drugs. In ordinary medical practice, doctors should avoid medical damage as long as they have sufficient duty of care, and the uncertainty of clinical trial risk requires that the duty of clinical trial, the content and standards of informed consent should be more stringent. 


\section{3 problems in the ethical review system of clinical trials}

The original intention of establishing the ethics committee is to provide a public guarantee for the rights and interests of the subject in clinical trials, to protect the rights and interests of the subject. The responsibility of the ethics committee is critical to ensure that clinical trials of drugs conform to ethical and scientific principles, but there are still problems in the independence and internal and external supervision of the ethics committee system in China ${ }^{[17]}$.

\subsubsection{The independence of the members of the ethics committee is not enough}

At present, most of the members of the ethics committee in our country are formed within the trial organization, and the proportion of the members is unknown. In the ethical review of clinical trials, it is inevitable to be influenced by the temptation of the trial organization's interests and the relationship between colleagues. Many ethical review committees and the trial organization are mostly dependent, so it is difficult to guarantee the neutrality of the review committee It is not conducive to the independent, fair and scientific development of the ethical review work, and the regulatory function of the ethics committee has not been fully played, which is not conducive to the protection of the rights and interests of the subject.

\subsubsection{The training and education mechanism of the ethics committee is not perfect}

Although the guiding principles for ethical review of drug clinical trials stipulates that the composition of the members of the ethics committee should include at least 5 persons with disciplinary background, the proportion of the members is not specified in detail, and the rationality of the structure of the members of the committee will have an important impact on the scientific and reasonable review results. The core of " the quality management standard of drug clinical trials" is ethics and science. The members of the ethics committee in China are generally experts in medicine, pharmacy and public health. Although these experts can evaluate the project from their professional perspective during the audit, on the other hand, they are too specialized due to lack of relevant ethical and legal knowledge They will be limited to make more just and comprehensive judgments. Therefore, it is necessary to strengthen the education and training of the members of the review committee and safeguard the legitimate rights and interests of the subject.

\subsubsection{Insufficient supervision of the review committee}

In China, the methods for ethical review of biomedical research involving human beings and the guiding principles for ethical review of drug clinical trials both regulate the system of ethical committee, which stipulates the supervision of health administrative departments at all levels, the supervision of medical ethics Expert Committee and the self-supervision of medical institutions, but in practice, the supervision of administrative departments does not stipulate how to start supervision from the perspective of internal supervision, China's ethics committee system is based on the western model, and some of its "sinicized" features are added. However, in the western model, the cornerstone of the system is individualism and liberalism, while China is an "acquaintance society", which pays attention to human feelings and feelings, so it is difficult for internal supervision to have a real supervision effect.

\section{Countermeasures and suggestions for improving the protection of subject's rights and interests}

The progress and development of medical research can not be separated from the important link of clinical trials. Improving the protection mechanism of the rights and interests of the subject in clinical trials can not only reduce the risk of the subject in clinical trials, protect the rights and interests of the subject, attract potential subject to join the trials, but also prevent the disputes between doctors and patients, thus promote the healthy development of clinical trials and improve the medicine in China research level.

\subsection{Improvement of legislation related to clinical trials}

\subsubsection{Issue clinical trial law to improve the level of legal norms}

The improvement of clinical trial legislation can make the common people eliminate their doubts about clinical trials, treat and participate in clinical trials correctly, and promote the progress and development of Chinese medicine and pharmacy. From the problems in the legislation of clinical trials in China, we can see that most of the legal norms applicable to clinical trials in China are administrative regulations, which have profound legal effects, limited scope of application and little impact on society. According to the provisions of the administrative punishment law of China, administrative rules can only make relevant punishment provisions within the scope of laws and administrative regulations. If there are no provisions in laws and administrative regulations, only a warning or a certain amount of fine can be imposed. That is to say, according to the administrative regulations on clinical trials in China, there is no legal sanction and criminal liability. The establishment of clinical experiment law to improve the level of the legal norms of clinical trials can also be reflected in the national level's attention to the norms of the clinical trial process, which is more conducive to the protection of the rights of subject in clinical trials ${ }^{[18]}$.

\subsubsection{Clear the rules and principles of tort liability in clinical trials}

The tort law of our country has not stipulated the tort of the clinical trial. We can learn from the relevant systems of other countries, improve the tort system of clinical trial and expand the tort liability clauses of the clinical trial in combination with the actual situation of our country. The difference between clinical trial and common medical treatment is described in the preceding article. Therefore, compared with common medical treatment, the trial Party of clinical trial The 
duty of informing, paying attention to and protecting subject was higher than that of ordinary medical behaviors. Therefore, in the tort liability law, the clinical trial tort liability is defined as a special tort, which clearly distinguishes the clinical trial tort liability and the general medical damage liability, to provide sufficient legal basis for the subject when their rights and interests are infringed ${ }^{[19]}$. The principle of presumption of fault should be applied to the principle of imputation of tort in clinical trials. That is to say that the trial party shall prove that there is no fault in the clinical trial, and the trial party shall also bear the burden of fault-proof. As the information between the experimenter and the experimenter is not equal, adopting the principle of fault presumption is conducive to lightening the burden of proof of the experimenter, improving the attention obligation of the experimenter in the course of the experiment, raising the vigilance, and motivating the experimenter to take effective risk prevention and control.

\subsubsection{Improve the relief system of the tester's damage}

From the ethical point of view, the clinical trial damage relief is justified, but in reality, there are still many problems in the construction of the clinical trial damage relief system. At present, we can use the independent clinical trial tort compensation system and the clinical trial compulsory insurance system of western countries for reference, and in special circumstances, the compulsory compensation and the independent compensation mechanism of the trial party as the supplementary risk dispersion mechanism, so as to establish the damage risk dispersion mechanism which adapts to China's national conditions. In clinical trials, the compensation of subject can be divided into two types: compulsory compensation and voluntary compensation. When applying for clinical trials, according to the specific items and risk levels of clinical trials, the sponsor is forced to define the compensation/compensation standard of subject in the research cost. Voluntary compensation can confirm the compensation principle, content and standard of the testing party by signing a contract.

Compared with the lengthy litigation, the insurance is convenient and fast, which shortens the time cost and litigation cost of the subject. Therefore, the establishment of clinical trial insurance system is one of the essential methods to disperse risk mechanisms. At present, the operability of clinical trial insurance in China is not reliable. Before the establishment of comprehensive trial insurance, the trial party can be forced to buy insurance for the subject. In case of damage to the subject caused only because of the experimental drug itself, the insurance compensation shall be started first when the damage occurs. For the part beyond the insurance, the sponsor and the trial party shall jointly bear the liability for compensation. The insurance system can ensure that the sponsor and the experimenter have enough financial ability to bear the responsibility to the experimenter.

\subsection{Suggestions on the protection of the right of informed consent of the subject}

The tested party shall fulfill the obligation of informed consent and information according to the standard that the subject is fully informed. First of all, in the object of notification, the object of notification is distinguished. When a subject is a person with full capacity for civil conduct, he/she is the object of informed consent; when a subject is a person without capacity for civil conduct or with limited capacity for civil conduct, the near relative, legal guardian or legal agent should be the object of notification. Special restrictions shall be made to the minors, people with mental health and other special groups to fully consider the interests of the subject and ensure that the rights and interests of the subject are fully protected. Secondly, the content of notification needs to be comprehensive and authentic. The tester shall inform the subject of all complete information and precautions related to the test, especially the possible risks during the test, remedial measures, alternative plans and test plans. At the same time, the subject should also be informed that they have the right to decide when to join or withdraw at any time during the trial. Finally, the content should be fully understood by the subject. Knowing is the premise, and we need to understand and then agree. The experimenter needs to explain the contents of the experiment in detail according to the different situations of the subject, especially the different risk levels of the experiment. The experimenter shall decide whether he/she is willing to participate in the experiment at his/her discretion, to ensure that he/she can fully exercise the right of informed consent.

\subsection{Strengthen internal and external supervision of the ethics review committee}

To ensure the effectiveness of supervision, we must start from the outside and ensure the legitimacy and neutrality of the review process, to obtain the fairness of the results. From the perspective of internal supervision, it is the first thing to realize the independence of the members of the ethical review committee. In line with the principles of independence, pluralism, openness and transparency, in terms of personnel composition, in addition to pharmaceutical, medical and public health experts, it should also include a certain proportion of psychology, ethics experts, subject and other personnel. At the same time, it is necessary to regularly train the medical, pharmaceutical and other experts in the review committee in terms of ethics and law. In terms of review content, in addition to the scientificity of the test plan, we should pay more attention to the integrity and comprehensiveness of the test project, highlight the interests of the subject, and evaluate the scientific value of the test based on considering various factors in an all-round way.

From the perspective of external supervision, first, set up the corresponding system from the perspective of administrative supervision, strengthen the access management of clinical trial applicants and the administrative supervision of specific trial work from the perspective of administrative supervisor, and protect the rights of the subject from the source by focusing on the access approval and adequate administrative supervision of clinical trials. Second, we should introduce the 
mechanism of external security system of administrative agencies and establish a unified clinical trial registration system. Under the condition of complete anonymity of subject participating in the trial, the registration system requires the trial party to disclose the trial progress and management information to the public. Through the registration platform, medical, ethical and legal experts, potential subject in the society, investors and ethics committee interested in the clinical trial can timely understand the trial information, strengthen the supervision of the trial process, and It helps to eliminate the misunderstanding of the public about the unknown clinical trials.

\section{Conclusion}

To sum up, the clinical trial is a necessary step before the new drug goes on the market. Only after the clinical trial can we find out the actual efficacy of a new drug on the human body. In order to ensure the effectiveness and safety of the new drug, the clinical trial has become an indispensable part. As an indispensable part of clinical trials, the subject' legal rights and interests should be put in the top priority because of the high risk and uncertainty of clinical trials and the relatively weak position of the subject. Based on the analysis of the problems existing in legislation, informed consent right and ethical review in the protection of the rights and interests of subject in China, this paper puts forward the ideas of protecting the rights and interests of subject and promoting the healthy development of clinical trials from the perspectives of improving legislation, improving the informed consent right of subject and strengthening the supervision of the ethical review committee, hoping to some extent promote the reasonable and legal development of clinical.

\section{References:}

[1] Li rui.Gao rui. Tang Xudong et al. Discussion on the management and operation mode of clinical research coordinators

[J]. Chinese Journal of New Drugs, 2012(21):2480-2484.

[2] Zhuo Hong. Su Binghua. Data management in double-blind clinical trials [J]. Chinese health statistics, 2000(1). 59-60.

[3] Yang Fan.Wang Mengyuan. Tao Tiantian et al. System of clinical trial liability insurance compensation for subjects in China [J]. Chinese Journal of New Drugs, 2016(16):1881-1885.

[4] SUN Tongbo, YIN Mei, Zhang Xue et al. Protect the Rights and Interests of the Subjects with the Clinical Trials of Drugs from the Legal Perspective [J]. Chinese hospital management, 2016, 36(4).73-74.

[5] Wang Xiaomin. (2012). Study on the ethical issues of randomized clinical trials. Chinese medical ethics, 25(4), 405-407.

[6] Xu Yunfeng. (2003). Several problems that need to be paid attention to by both doctors and patients in implementing the right to know. China Health Care Management, 19(6), 349-349.

[7] Cui Yuming.Cai Zhijiu. Thoughts on the privacy of patients with diseases [J]. China Health Care Management, 2003, 19(10):607-608.

[8]Ji Tao. Who is the subject of informed consent in medical relations? [J]. Zhejiang Social Sciences, 2010(2):10-13.

[9]Liu Ang. The Historical Change of the Informed Consent and Legal Enlightenment [J]. Hebei Law Science, 2015, 33(4):176-183.

[10]Du Lifu.Jin YI. Study on the privacy of patients in the relationship between doctors and patients [J]. Contemporary Law Review, 2001(7):79-80.

[11] Zeng Lingfeng.Liu Jun.Pan Jianke. Study on the ethical issues of vulnerable groups and rights protection in drug clinical trials [J]. Chinese Journal of New Drugs, 2016(24):2793-2798.

[12] Zhang Qing.Sun Yikang. Current status and administration for drug clinical trial institutions in Shanghai [J]. Chinese Journal of New Drugs and Clinical Medicine, 2009(6):469-471.

[13] Wang Yue. Rethinking the Legal Issues in China's Drug Clinical Trials from the "Korea Ginseng Pill Event" [J]. Chinese pharmacy, 2005, 16(10):724-727.

[14] Zheng Yifei.Jiang Baisheng.Zhang Fumin. Discussion on the Connotation and Protection of the Right of Informed Consent in Drug Clinical Trials [J]. China Health Care Management, 2011, 28(4):278-279.

[15] Zhang Wei.Xiong Hongyan.Deng Qiangting. Ethical surveys and case studies involving animal experiments and clinical trials in medical papers [J]. Chinese Journal of Science and Technology Periodical, 2017, 28(4):300-305. 
[16] Wang Jiesong.Liu Gang.Wu Jiuhong. Risk and management of drug clinical trials [J]. Chinese pharmacy, 2008(31):2406-2407.

[17] Wen Ke.Wang Jin.Bai Nan. Discussion on the management of subject risk minimization in drug clinical trials [J]. Chinese Journal of New Drugs, 2015(16):1862-1866.

[18] Yan Yongbo.Li Ye. Comparative Study on Chinese Drug Clinical Trial Regulations and Relevant International Regulations [J]. Chinese Journal of New Drugs, 2011(17):1612-1614.

[19] Liu Xuemin. Civil law image of informed consent of clinical trial subjects [J]. Journal of Hubei University of Police, 2014, 27(8):104-108. 